\title{
RESONANT SUBBAND-LANDAU LEVEL COUPLING IN SYSTEMS WITH ANISOTROPIC AND TILTED VALLEYS*
}

\author{
M. ZALUŻNY \\ Institute of Physics, M. Curie-Skłodowska University \\ Pl. M. Curie-Skłodowskiej 1, 20-031 Lublin, Poland
}

AND W. OKULSKI

Physics Department of Medical Academy, ul. Cicha 4, 20-078 Lublin, Poland

The problem of the anticrossing of the Landau levels originating from the ground subband and first excited subband in the quasi-two-dimensional systems with anisotropic valleys in the presence of the tilted magnetic field is discussed in the framework of the perturbation theory. The full field coupling regime is considered. Comparison with the existing theoretical and experimental results is given.

PACS numbers: 73.20.Dx

The purpose of this note is to give a simple perturbative description of the resonant subband-Landau splitting in the quasi-two-dimensional system with anisotropic valleys when simultaneously the magnetic field $(B)$ and the valley is tilted (with respect to the surface normal $n$ ). The case $B \| n$ is discussed theoretically in [1] and studied experimentally in the $n$-type inversion layer on (118) $\mathrm{Si}$ surface by Cheng and McCombe [2].

When the valley is tilted it is convenient to transform the standard effective mass Hamiltonian $(\mathcal{H})$ into the following form $[1,3,4]$ :

$$
\tilde{\mathcal{H}}=S^{-1} \mathcal{H} S=\tilde{\mathcal{H}}_{0}+\tilde{\mathcal{H}}_{1}+\tilde{\mathcal{H}}_{2}
$$

with

$$
\tilde{\mathcal{H}}_{0}=\tilde{\mathcal{H}}_{\|}+\tilde{\mathcal{H}}_{\perp}=\left(P_{x}^{\prime 2}+p_{y}^{\prime 2}\right) / 2 m_{\mathrm{d}}+\left(p_{z}^{2} / 2 m_{z}+V(z)\right)
$$

*This work is supported in part by the Ministry of National Education under contract No II. 1.9.P/04/063. 


$$
\begin{aligned}
& \tilde{\mathcal{H}}_{1}=\left[\left(\tan \phi_{y}-\gamma\right) K_{x}^{2}\left(K_{y} / K_{x}\right)^{1 / 2} P_{x}^{\prime}-\tan \phi_{x} K_{y}^{2}\left(K_{x} / K_{y}\right)^{1 / 2} p_{y}^{\prime}\right] \frac{\hbar z}{m_{\mathrm{d}} l^{2}} \\
& \tilde{\mathcal{H}}_{2}=\left[\left(\tan \phi_{y}-\gamma\right)^{2} K_{x}^{2}+\left(\tan \phi_{x} K_{y}\right)^{2}\right] \frac{(\hbar z)^{2}}{2 m_{\mathrm{d}} l^{4}}
\end{aligned}
$$

where $\stackrel{\alpha}{\alpha}=\stackrel{\leftrightarrow}{m}^{-1}$ is the reciprocal effective mass tensor, $V(z)$ is the layer potential, $\phi_{y}=\arctan \left(B_{y} / B_{z}\right), \phi_{x}=\arctan \left(B_{x} / B_{z}\right), S=\exp \left(-\mathrm{i} \gamma\left(z p_{y}-z^{2} e B_{x} / 2 c\right) / \hbar\right)$, $P_{x}^{\prime}=\left(p_{x}^{\prime}-\hbar y^{\prime} l^{-2}\right), \gamma=\alpha_{y z} / \alpha_{z z}, l^{2}=c \hbar / e B_{z}, m_{x}^{-1}=\alpha_{x x}, m_{z}^{-1}=\alpha_{z z}, m_{y}^{-1}=$ $m_{y y}^{-1}=\alpha_{y y}-\alpha_{y z}^{2} / \alpha_{z z}, m_{\mathrm{d}}=\left(m_{x} m_{y}\right)^{1 / 2}, K_{i}=\left(m_{\mathrm{d}} / m_{i}\right)^{1 / 2}, x^{\prime}=x / K_{x}, y^{\prime}=$ $y / K_{y}, p_{x}^{\prime}=p_{x} K_{x}$, and $p_{y}^{\prime}=p_{y} K_{y}$. We use such coordinate system that $\alpha_{x z}=$ $\alpha_{x y}=0$ and employ the gauge in which the vector potential of the magnetic field $\boldsymbol{B}=\left(B_{x}, B_{y}, B_{z}\right)$ is equaled $\boldsymbol{A}=\left(B_{y} z-B_{z} y,-B_{x} z, 0\right)$. The spin-dependent term in (1) is neglected for simplicity.

The Landau $(|M\rangle)$ and subband $(|N\rangle)$ levels are the exact eigenfunctions of $\tilde{\mathcal{H}}_{\|}$and $\tilde{\mathcal{H}}_{1}$, respectively. The eigenvalues of $\tilde{\mathcal{H}}_{0}$ are given by $E_{N M}^{(0)}=E_{N}+$ $(M+1 / 2) \hbar \omega_{\mathrm{c}}^{2 \mathrm{D}}$, where $E_{N}$ is the energy of the $N$-th electric subband when $B=0$ and $\hbar \omega_{\mathrm{c}}^{2 \mathrm{D}}=\hbar e B_{z} / \mathrm{cm} m_{d}$.

The second term in (1) couples the levels $|N, M\rangle$ and $\left|N^{\prime}, M \pm 1\right\rangle$ removing the degeneracy at the crossover i.e. when the subband separation $E_{10}=E_{1}-E_{0}$ is close to the cyclotron energy $\hbar \omega_{\mathrm{c}}^{2 \mathrm{D}}$. The first-order perturbation theory for the degenerate levels $|0.1\rangle$ and $|1.0\rangle$ yields the following formula for the normalized level splitting $\left(\Delta \mathcal{E}=\Delta E / E_{10}\right)[1]$ :

$$
\Delta \mathcal{E}=2\left|\left\langle 0.1\left|\tilde{\mathcal{H}}_{1}\right| 1.0\right\rangle\right| / E_{10}=f_{10}^{1 / 2} \varepsilon
$$

Here $f_{10}=2|\langle N=1|z| N=0\rangle|^{2} E_{10} m_{z} / \hbar^{2}$ is the oscillator strength, $\varepsilon^{2}=$ $\left[\varepsilon_{1}-\tan \phi_{y}\left((\mathcal{K}-1) \sin ^{2} \varphi+1\right) / \mathcal{K}^{1 / 2}\right]^{2}+\left(\tan \phi_{x}\right)^{2}\left(\left(\mathcal{K}^{-1}-1\right) \cos ^{2} \varphi+1\right)$ with $\varepsilon_{1}=\left(\mathcal{K}+\mathcal{K}^{-1}-2\right)^{1 / 2} \sin \varphi \cos \varphi$, where $\varphi$ is the angle between $n$ and the principal axes of the valley $(C), \mathcal{K}=m_{l} / m_{t}, m_{l, t}$ are the principal values of the effective mass tensor $\stackrel{\leftrightarrow}{m}$. (From the above equation we can conclude that perturbed approach used in this paper is in principle correct when $\varepsilon \ll 1$.)

When $B \| n$ then $\varepsilon=\varepsilon_{1}$ and the energy splitting resulting from (5) coincides with that obtained in [1].

In the case of the inversion layer on the Si surface the potential $V(z)$ can be approximated by the triangular well with infinite barrier at the surface. The above approximation gives $f_{10}^{1 / 2} \cong 0.856$. Taking $\left.\mathcal{K}\right|_{\mathrm{Si}}=4.8$ we find that for the layer on the (118) Si surface $\left(\varphi=10^{\circ}\right)$ the energy splitting is equaled $\Delta \mathcal{E} \cong 0.267$. This value is slightly smaller $(\approx 20 \%)$ than that obtained in experiment [2]. (The discrepancy is probably connected with anomalous behavior of cyclotron energy at low electron density abserved in the Si $n$-type inversion layers [2].) When $\varepsilon \ll 1$ (like in the system studied in [2]) the main difference between our result and that obtained in the framework of the Drude model [2] lies in the absence of the factor $f_{10}^{1 / 2}$ in the last one. Note that this factor is sensitive to the form of the layer potential $V(z)$ and can deviate significantly from unity particularly in the case of 


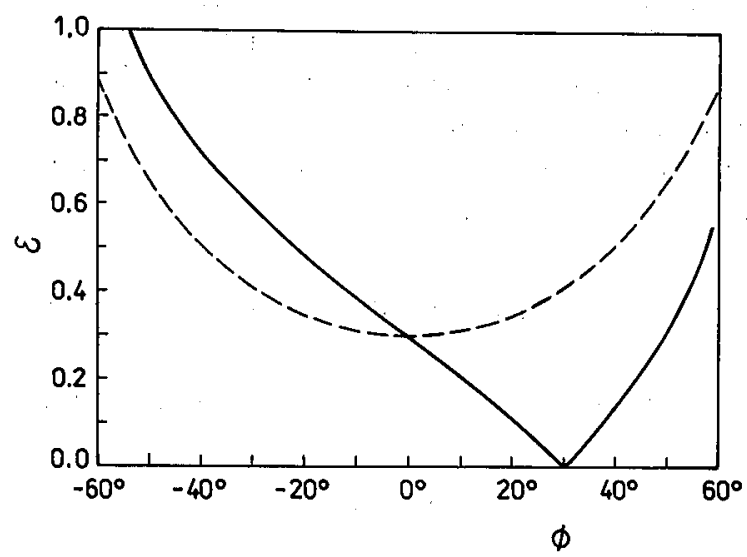

Fig. 1. The dependence of the parameter $\varepsilon$ on the tilt angle $\phi$ in the layer on the (118) Si surface. (-) result obtained for $B$ laying in the $y-z$ plane, $(---)$ result obtained for $B$ laying in the $x-z$ plane. $\left.\mathcal{K}\right|_{s i}=4.8, \varphi=10^{\circ}$.

accumulation layers. This indicates that theory developed by the authors of Ref. [2] is too simplified to be used for quantitative interpretation of experiments.

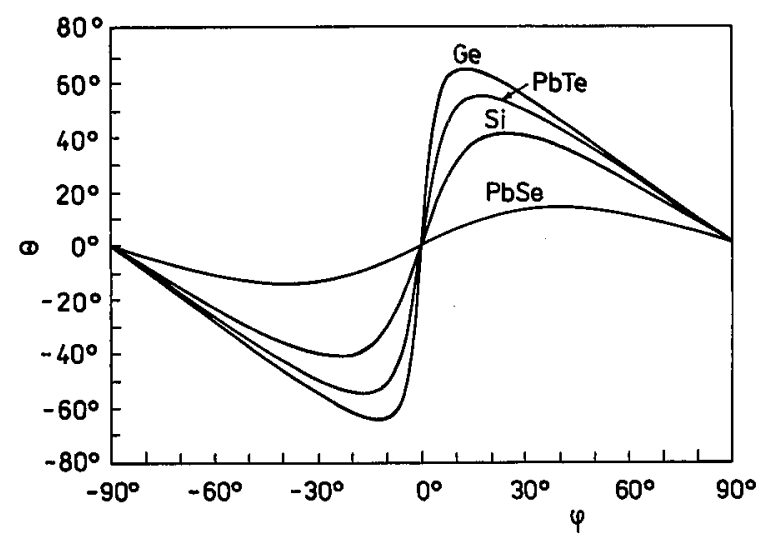

Fig. 2. The dependence of the angle $\theta=\arctan \gamma$ on the tilt angle $\varphi$ in $\mathrm{Ge}\left(\left.\mathcal{K}\right|_{\mathrm{Ge}}=\right.$ 19.5), $\mathrm{PbTe}\left(\left.\mathcal{K}\right|_{\mathrm{PbTe}}=10\right), \mathrm{Si}\left(\left.\mathcal{K}\right|_{\mathrm{Si}}=4.8\right)$ and $\mathrm{PbSe}\left(\left.\mathcal{K}\right|_{\mathrm{PbSe}}=1.6\right)$.

When $\varphi \neq 0$ the strength of the subband-Landau level coupling depends in more complicated way on orientation of the magnetic field than in the case $\varphi=0$ [5]. Figure 1 shows the variation of the parameter $\varepsilon$ with $\phi(=$ the angle between $n$ and $B$ ) in the layer on the (118) Si surface. Particularly interesting is the configuration when vectors $B, C$ and $n$ lie in the same plane $\left(\phi_{x}=0, \phi_{y}=\phi\right)$. Then, for $\phi=\theta \equiv \arctan \gamma$ the Hamiltonian $\tilde{\mathcal{H}}$ coincides with $\tilde{\mathcal{H}}_{0}$ and parameter 
$\varepsilon$ vanishes i.e. the coupling induced by the tilted configuration of the valley is compensated by the effects induced by the tilted configuration of the magnetic field. The variation of the angle $\theta$ with $\varphi$ in some materials is shown in Fig. 2.

We think that the electrons on the (118) Si surface studied experimentally in Ref. [2] should be good candidates for the observation of the disappearance of the level splitting predicted in this paper.

\section{Acknowledgements}

We thank J. Arseniuk for performing the numerical calculations.

\section{References}

[1] M. Załużny, W. Okulski, Acta Phys. Pol. A71, 173 (1987).

[2] J.-P. Cheng, B.D. McCombe, Phys. Rev. B 38, 10974 (1988).

[3] F. Stern, W.E. Howard, Phys. Rev. 163, 816 (1967).

[4] W. Okulski, M. Załużny, Semicond. Sci. Technol. 6 (in press).

[5] M. Załużny, Phys. Rev. B 40, 8495 (1989). 